\title{
A high affinity human monoclonal antibody against Pfs230 binds multiple parasite stages and blocks oocyst formation in mosquitoes
}

Camila H. Coelho',11, Wai Kwan Tang2,11, Martin Burkhardt ${ }^{3}$, Jacob D. Galson ${ }^{4,5}$, Olga Muratova ${ }^{3}$, Nichole D. Salinas ${ }^{2}$, Thiago Luiz Alves e Silva ${ }^{6}$, Karine Reiter ${ }^{3}$, Nicholas J. MacDonald ${ }^{3}$, Vu Nguyen ${ }^{3}$, Raul Herrera ${ }^{3}$, Richard Shimp ${ }^{3}$, David L. Narum ${ }^{3}$, Miranda Byrne-Steele ${ }^{7}$, Wenjing Pan ${ }^{7}$, Xiaohong $\mathrm{Hou}^{7}$, Brittany Brown ${ }^{7}$, Mary Eisenhower ${ }^{7}$, Jian $\operatorname{Han}^{7}$, Bethany J. Jenkins ${ }^{1}$, Justin Yai Alamou Doritchamou ${ }^{1}$, Margery G. Smelkinson ${ }^{8}$, Joel Vega-Rodriguez ${ }^{6}$, Johannes Trück ${ }^{4}$, Justin J. Taylor ${ }^{9}$, Issaka Sagara ${ }^{10}$, Jonathan P. Renn ${ }^{3}$, Niraj H. Tolia ${ }^{2,12^{*}}$, Patrick E. Duffy ${ }^{1,12^{*}}$

1 - Pathogenesis and Immunity Section, Laboratory of Malaria Immunology and Vaccinology, National Institute of Allergy and Infectious Diseases, National Institutes of Health, Bethesda, MD, USA 2 - Host-Pathogen Interactions and Structural Vaccinology Section, Laboratory of Malaria Immunology and Vaccinology, National Institute of Allergy and Infectious Diseases, National Institutes of Health, Bethesda, MD, USA

3- Vaccine Development Unit, Laboratory of Malaria Immunology and Vaccinology, National Institute of Allergy and Infectious Diseases, National Institutes of Health, Bethesda, MD, USA

4 - University Children's Hospital, and the Children's Research Center, University of Zurich, Zurich, Switzerland

5- Alchemab Therapeutics Ltd, 55-56 Russell Square, London, WC1B 4HP, UK 6 - Laboratory of Malaria and Vector Research, National Institute of Allergy and Infectious Diseases, National Institutes of Health, Rockville, MD, United States 7 - iRepertoire Inc., Huntsville, AL, USA.

8- Biological Imaging Section, National Institute of Allergy and Infectious Diseases, National Institutes of Health, Bethesda, MD, USA

9 - Fred Hutchinson Cancer Research Center, Seattle, WA, USA

10 - Malaria Research and Training Center, University of Sciences, Techniques, and Technology, Bamako, Mali 11-These authors contributed equally: Camila H. Coelho, Wai Kwan Tang 12- These authors jointly directed this work Niraj H. Tolia1, Patrick E. Duffy

${ }^{*}$ Corresponding authors:

patrick.duffy@nih.gov and niraj.tolia@nih.gov

Laboratory of Malaria Immunology and Vaccinology, National Institute of Allergy and Infectious Diseases, National Institutes of Health, 9000 Rockville Pike, Bethesda MD 20892, USA. 
43

44

45

46

47

48

49

50

51

52

53

54

55

56

57

58

59

60

61

62

63

64

65

66

3

65

6

\section{ABSTRACT}

Malaria elimination requires tools that interrupt parasite transmission. Here, we characterized B cell receptor responses among Malian adults vaccinated against the first domain of the cysteine-rich $230 \mathrm{kDa}$ gamete surface protein $\mathrm{Pfs} 230^{1-3}$ to neutralize sexual stage $P$. falciparum parasites and halt their further spread. We generated nine Pfs230 human monoclonal antibodies (mAbs). One mAb potently blocked transmission to mosquitoes in a complement-dependent manner and reacted strongly to gamete surface while eight mAbs showed only low or no blocking activity. This study provides a rational basis to improve malaria vaccines and develop therapeutic antibodies for malaria elimination.

5

6

7

8

9

60

1

4




\section{MAIN TEXT}

Malaria eradication is a global priority and will require innovative strategies that, in addition to preventing or controlling human infection, might block parasite transmission through mosquitoes. Sequences of matched heavy and light chain variable regions from single human $B$ cells have been used to identify antibodies generated in response to infection or vaccination and inform vaccinology $y^{4-7}$. In this study, we apply this approach to examine human antibodies elicited in response to a transmission blocking vaccine (TBV), that used a Pfs230 fragment as antigen. Pfs230 is present on the surface of $P$. falciparum gametocytes and gametes and mediates binding of exflagellating microgametes to red blood cells, thus parasites lacking this protein cannot bind to red blood cells or further develop into oocysts. ${ }^{1}$ We collected Pfs230 domain 1 (D1)-specific single memory B cells (Extended Data Fig. 1, Extended Data Fig. 2a) from eight Malian adults immunized with four doses of Pfs230D1-EPA/Alhydrogel ${ }^{\circledR}$ vaccine (Clinicaltrials.gov NCT02334462) to identify functional monoclonal antibodies elicited in response to a TBV. This vaccine aims to neutralize sexual stage $P$. falciparum parasites by targeting Pfs230, a 230kDa gamete surface protein comprised of fourteen 6-cysteine (6-Cys) domains ${ }^{1-3}$. All samples were chosen from subjects presenting high serum Transmission-Reducing Activity (TRA), measured by the capacity of serum antibodies from immunized subjects to reduce the number of oocysts that develop in mosquitoes fed on in vitro cultured $P$. falciparum gametocytes (Extended Data Table 1).

We obtained $272 \mathrm{VH}$ and $351 \mathrm{VL}$ sequences of $\mathrm{B}$ cell receptor (BCR) from Pfs230D1-specific single memory B cells from the vaccinees via amplification and 
sequencing of the $V(D) J$ region (Extended Data Fig. 3). When analysing $V$ gene usage of the BCR sequences, $87.5 \%$ of the subjects presented Pfs230D1-specific memory B cells using kappa chains derived from IGKV4-1 (Extended Data Fig. 2e). This light chain gene has also been identified in sequences of functional human mAbs obtained in response to other Plasmodium antigens ${ }^{4-6,8}$. For the heavy chain, IGHV1-69 was the most commonly expressed gene and detected in 100\% (8/8) of vaccinees (Extended Data Fig. 2f). IGHV1-69 is one of the most polymorphic loci of the IGHV gene cluster ${ }^{9}$ and is frequently found in broadly neutralizing antibodies generated in response to influenza haemagluttinin ${ }^{10,11}$.

Nine pairs of BCR sequences were chosen for expression of fully human Pfs230D1 IgG1 antibodies by assessing whether the CDR3 sequences were shared between sorted B cells. This approach identifies identical sequences in multiple B cells from the same subject, indicating that they have been activated in response to vaccination. These nine pairs (Fig. 1a) represented distinct heavy and light chain germline genes with an overabundance of IGHV1-18 (N=6), IGHV1-69 (N=3), and IGKV4-1 ( $\mathrm{N}=7)$. The resulting recombinant antibodies bound to Pfs230D1 antigen (Figure 1d,e, Extended Data Figure 4). Competitive epitope binning of the nine mAbs suggested they bind three non-overlapping epitopes in Pfs230D1 (Fig. 1b). LMIV230-01 forms a distinct group (Group 1) and has potent neutralizing activity (Fig. 1b, c). The remaining mAbs do not compete with LMIV230-01 and may form two additional epitope groups. Group 2 and 3 mAbs possess low or no neutralizing activity (Fig. 1c). We 
111 therefore focused most of our subsequent analyses on LMIV230-01 and to a lesser extent on LMIV230-02, which demonstrated low functional activity. strong and similar binding affinities (Fig. 1e, Extended Data Fig. 4, Extended Data Table 2). We confirmed the two mAbs bind distinct epitopes using competition ELISA (Extended data Figure 5d) consistent with the epitope binning results (Fig. 1b). Despite their shared use of IGHV1-69, LMIV230-01 and LMIV230-02 displayed numerous differences in their heavy chain CDRs, consistent with their recognition of distinct epitopes (Extended Data Figure 12).

Although presenting similar affinity to Pfs230D1, the mAbs differed in their functional activity as measured by SMFA. LMIV230-01 ablated $P$. falciparum oocyst burden in mosquitoes in a dose-dependent manner with $91.7 \%$ neutralization (TRA) at $1000 \mu \mathrm{g} / \mathrm{mL}$ (Fig. 1f). Importantly, 80.3\% neutralization was retained at $60 \mu \mathrm{g} / \mathrm{mL}$. On the other hand, LMIV230-02 reduced oocyst burden by only $58.7 \%$ at the maximum concentration of $1000 \mu \mathrm{g} / \mathrm{mL}$ and activity was lost at $250 \mu \mathrm{g} / \mathrm{mL}$. As previously reported, TRA values higher than $80 \%$ are highly reproducible across independent experiments ${ }^{12,13}$.Combining the two antibodies did not increase their overall activity: TRA values were not statistically different when 500 $\mu \mathrm{g}$ of LMIV230-02 was combined with $10 \mu \mathrm{g}$ of LMIV230-01 $(\mathrm{TRA}=58.7 \%)$ versus $10 \mu \mathrm{g}$ of LMIV230-01 alone (TRA= 52.5\%) in mosquito feeding assays (Extended Data Figure 5e). assessed binding to the native protein. Both mAbs reacted to the protein extract of 
133 parasites and were sensitive to reduction of the two disulfide-bonds, suggesting the

134 presence of conformational epitopes (Fig. 1g, Extended Data Fig. 5c). Interestingly,

135 LMIV230-01 strongly labelled the surface of live $P$. falciparum gametes purified 2 hours

136 post-exflagellation, while LMIV230-02 did not (Fig. 1h). This suggests that the

137 LMIV230-02 epitope is not completely accessible on the surface-displayed native

138 protein, possibly due to structural limitations imposed by the multi-domain protein

139 Pfs230, as has been seen for other proteins ${ }^{14,15}$ including another 6-Cys TBV

140 candidate $^{16}$. 
bioRxiv preprint doi: https://doi.org/10.1101/2020.09.25.313478; this version posted September 26, 2020. The copyright holder for this preprint (which was not certified by peer review) is the author/funder. All rights reserved. No reuse allowed without permission.

a

\begin{tabular}{lrl}
\multicolumn{1}{c}{ mAb } & $\begin{array}{c}\text { Heavy } \\
\text { chain }\end{array}$ & $\begin{array}{l}\text { Light } \\
\text { chain }\end{array}$ \\
LMIV230-01 & IGHV1-69 & IGKV1-5 \\
LMIV230-02 & IGHV1-69 & IGKV4-1 \\
GKV16 & IGHV1-69 & IGKV4-1 \\
GKV08 & IGHV1-18 & IGKV4-1 \\
GKV01 & IGHV1-18 & IGKV4-1 \\
GKV07 & IGHV1-18 & IGKV4-1 \\
GKV05 & IGHV1-18 & IGKV4-1 \\
GKV06 & IGHV1-18 & IGKV4-1 \\
GKV22 & IGHV1-18 & IGKV2-28
\end{tabular}

b

\begin{tabular}{|c|c|c|c|c|c|c|c|c|c|}
\hline \multirow[b]{3}{*}{ LMIV230-01 } & \multirow{2}{*}{$\frac{\text { Group } 1}{\text { LMIV230-01 L }}$} & \multicolumn{6}{|c|}{ Group 2} & \multicolumn{2}{|c|}{ Group 3} \\
\hline & & LMIV230-02 & GKV01 & GKV05 & GKV06 & GKV07 & GKV22 & GKV08 & GKV16 \\
\hline & & 95 & 100 & 100 & 99 & 100 & 100 & 100 & 100 \\
\hline LMIV230-02 & 85 & & 10 & 13 & 14 & 18 & 4 & 51 & 60 \\
\hline GKV01 & 100 & 0 & & 1 & 1 & 1 & 3 & 41 & 50 \\
\hline GKV05 & 100 & 0 & 0 & & 0 & 0 & 0 & 40 & 49 \\
\hline GKV06 & 100 & 0 & 0 & 0 & & 1 & 0 & 40 & 51 \\
\hline GKV07 & 100 & 0 & 0 & 0 & 1 & & 0 & 41 & 53 \\
\hline GKV22 & 89 & 0 & 0 & 4 & 3 & 7 & & 38 & 41 \\
\hline GKV08 & 71 & 0 & 0 & 0 & 0 & 0 & 0 & & 18 \\
\hline GKV16 & 24 & 0 & 0 & 0 & 0 & 0 & 0 & 0 & \\
\hline
\end{tabular}

\begin{tabular}{|c|c|c|c|c|c|c|c|c|c|c|}
\hline C & & LMIV230-01 & LMIV230-02 & GKV01 & GKV05 & GKV06 & GKV07 & GKV22 & GKV08 & GKV16 \\
\hline & $375 \mu \mathrm{g} / \mathrm{mL}$ & --- & & $20 \%$ & $0 \%$ & $10 \%$ & $10 \%$ & $0 \%$ & $0 \%$ & $0 \%$ \\
\hline & $250 \mu \mathrm{g} / \mathrm{mL}$ & $85 \%$ & $51 \%$ & --- & --- & --- & --- & --- & --- & --- \\
\hline
\end{tabular}

d

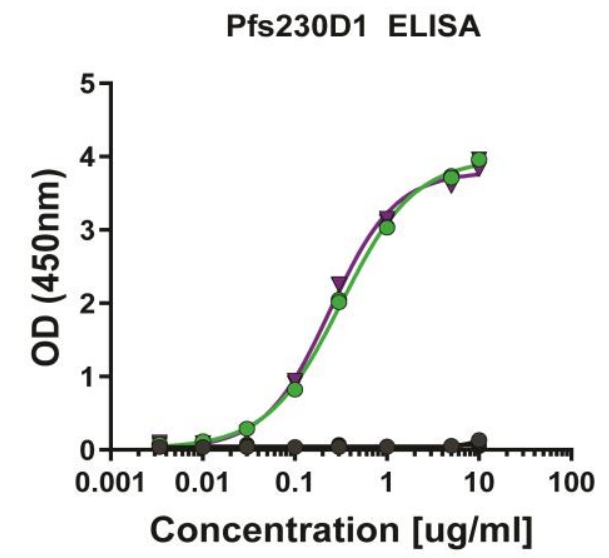

- LMIV230-01 - Pfs25 mAb

- LMIV230-02 - IgG1 isotype control e
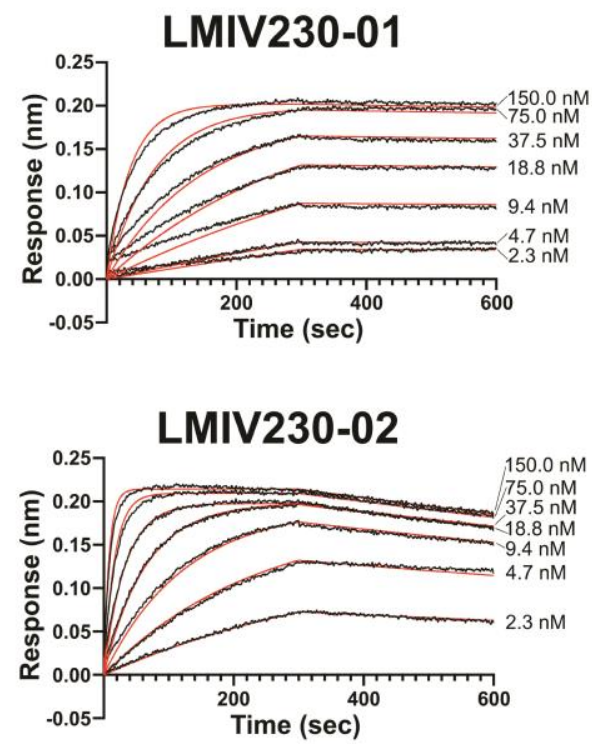

f

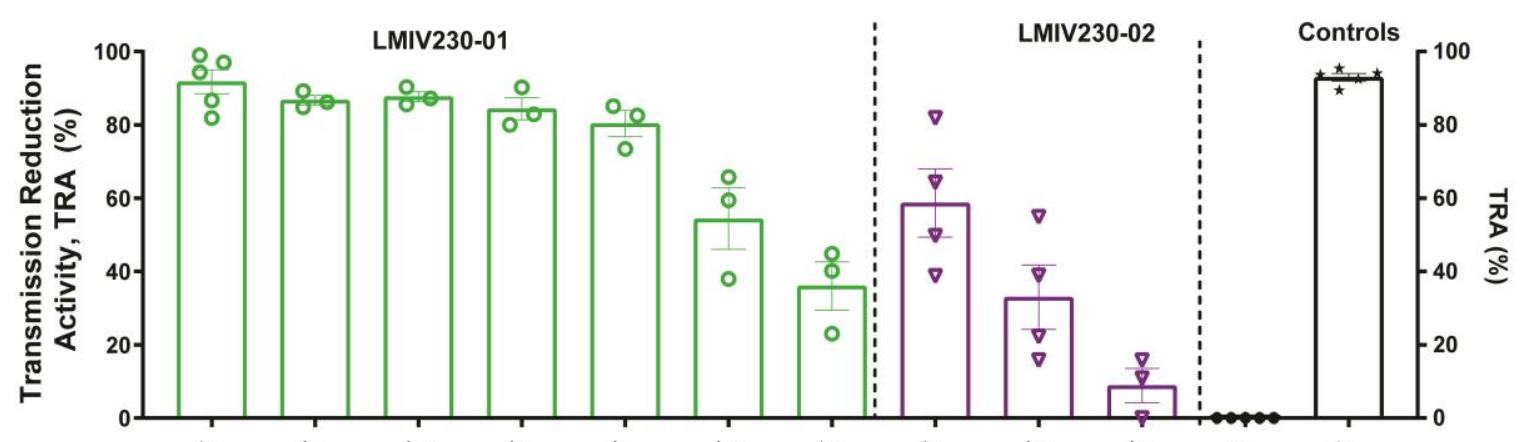

g

142

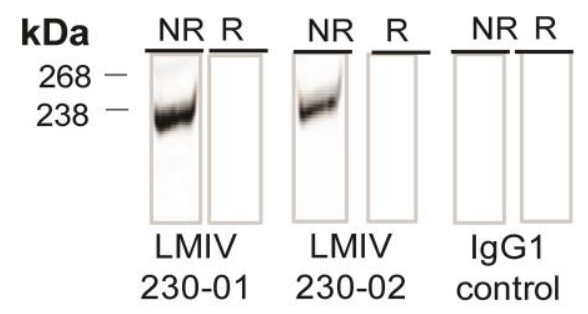

h

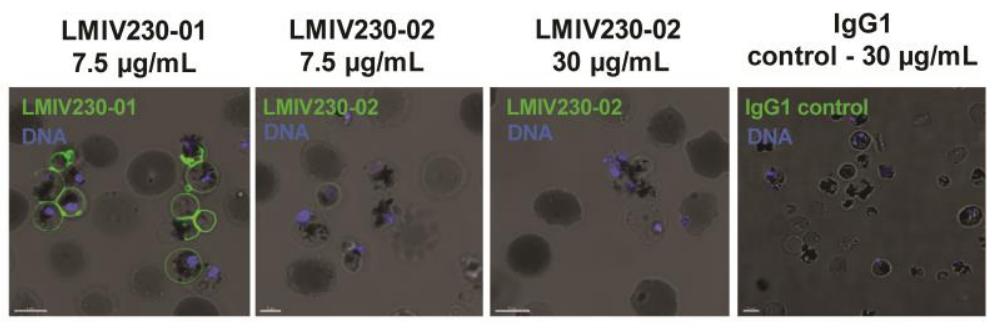


Fig. 1| Human recombinant mAbs were generated from Pfs230D1-specific single memory B cells of Malian adults vaccinated with the Pfs230D1-EPA/Alhydrogel ${ }^{\circledR}$ TBV. a, VH and VL genes corresponding to each mAb. LMIV230-01 and LMIV230-02 sequences originate from the IGHV1-69 heavy chain gene but utilize different kappa chain genes. Complete $\mathrm{V}$ gene usage determined in Pfs230-specific memory B cells is described in Extended Data Figures 2e,f. b, Epitope binning of human antiPfs230D1scFvs. The primary binding scFv is listed on the left and the competing scFv are listed on the top. Reported scores are a percentage of total binding of that antibody in the absence of a competitor scFv. Values greater that $50 \%$ display low amounts of competition, while values lower than $50 \%$ exhibit greater competition. Any experiment with $>100 \%$ binding was given a score of 100 , while negative values were given a score of 0 . Potential epitope bins are grouped and labelled above the table. c, Functional activity of each mAb, assessed by Standard Membrane Feeding Assay (SMFA) and measured as the \% reduction (versus control $\mathrm{mAb}$ ) in the number of $P$. falciparum NF54 oocysts in midguts of Anopheles mosquitoes ("TRA"). d, LMIV230-01 and LMIV230-02 mAbs bound similarly to Pfs230D1 and e, show high affinity to recombinant Pfs230D1 (Extended Data Fig. 4, Extended Data Table 2) f, LMIV230-01 reduces $P$. falciparum NF54 oocyst numbers by $91.7 \%$ at $1000 \mu \mathrm{g} / \mathrm{mL}, 86.7 \%$ at $500 \mu \mathrm{g} / \mathrm{mL}, 84.4 \%$ at $250 \mu \mathrm{g} / \mathrm{mL}$ and $80.3 \%$ at $60 \mu \mathrm{g} / \mathrm{mL}$, while LMIV230-02 displays only modest activity with $58.7 \%$ reduction at the maximum concentration of $1000 \mu \mathrm{g} / \mathrm{mL}$, in SMFA. Data from eleven independent SMFA and each concentration was evaluated in at least three biological replicates for each $\mathrm{mAb}$. $\mathrm{N} \geq 20$ mosquitos per assay. Average oocyst numbers in the control mosquitoes (fed with mouse lgG1 mAb targeting $P$. yoelii P140 protein) for each experiment were: $\exp .1=29.73$; exp. $2=7.18$; exp. 3= 57.86; exp. 4= 36.41; exp. $5=51.71$, exp. $6=4.55$; exp. $7=62.35$; exp. $8=20.50$, exp. $9=8.71$, exp $10=18.05$, exp. $11=5.86$. Negative oocyst reduction values were set to zero. Human isotype IgG1 and US human serum pool were used as additional negative controls (Extended Data Fig. 5b). Values are shown as mean \pm s.e.m. g, LMIV230-01 and LMIV230-02 bind to non-reduced (NR) protein extract of $P$. falciparum NF54 gametes purified on Nycodenz after 2 hours in exflagellation medium. $\mathbf{h}$, LMIV230-01 strongly binds to gametes at $7.5 \mu \mathrm{g} / \mathrm{mL}$ while LMIV230-02 does not bind at $7.5 \mu \mathrm{g} / \mathrm{mL}$. or $30 \mu \mathrm{g} / \mathrm{mL}$. Both mAbs were labelled with Alexa Fluor 488. Scale bars: $5 \mu \mathrm{M}$.

$$
\text { including gametocytes, exflagellation centers, microgametes, macrogametes and round }
$$

forms (zygotes) collected 4 hours after mosquito feeding. As expected, the mAb did not bind to the post-fertilization stage ookinetes, obtained 24 hours after the mosquito bloodmeal (Fig. 2a).

Pfs230 antibody activity depends on complement fixation to lyse $P$. falciparum ${ }^{17}$. To test whether the activity of LMIV230-01 was dependent on activation of the complement system, we incubated parasites with LMIV230-01 in the presence of intact or heat-inactivated sera from US donors then assessed lysis of gametes (Fig. 2b) as well as transmission of parasites fed to mosquitoes after treatment using the same 
181 conditions (Fig. 2c). Functional activity of LMIV230-01 to lyse gametes and block oocyst 182 formation in mosquitoes was substantially reduced in the heat-inactivated sera (Figs.

$1832 \mathrm{~b}$ and $2 \mathrm{c}$ ), demonstrating complement-dependency. Activation of complement leads to 184 the formation of the membrane attack complex (MAC), an assembly of the complement 185 molecules C5b, C6, C7, C8, and C918,19 on the parasite surface. Using an antibody that 186 recognizes assembled MAC, we demonstrated complement fixation on the surface of 187 live $P$. falciparum gametes that were incubated with LMIV230-01 in the presence of 188 intact but not heat-inactivated serum (Fig. 2d and Extended Data Fig. 7). 
a
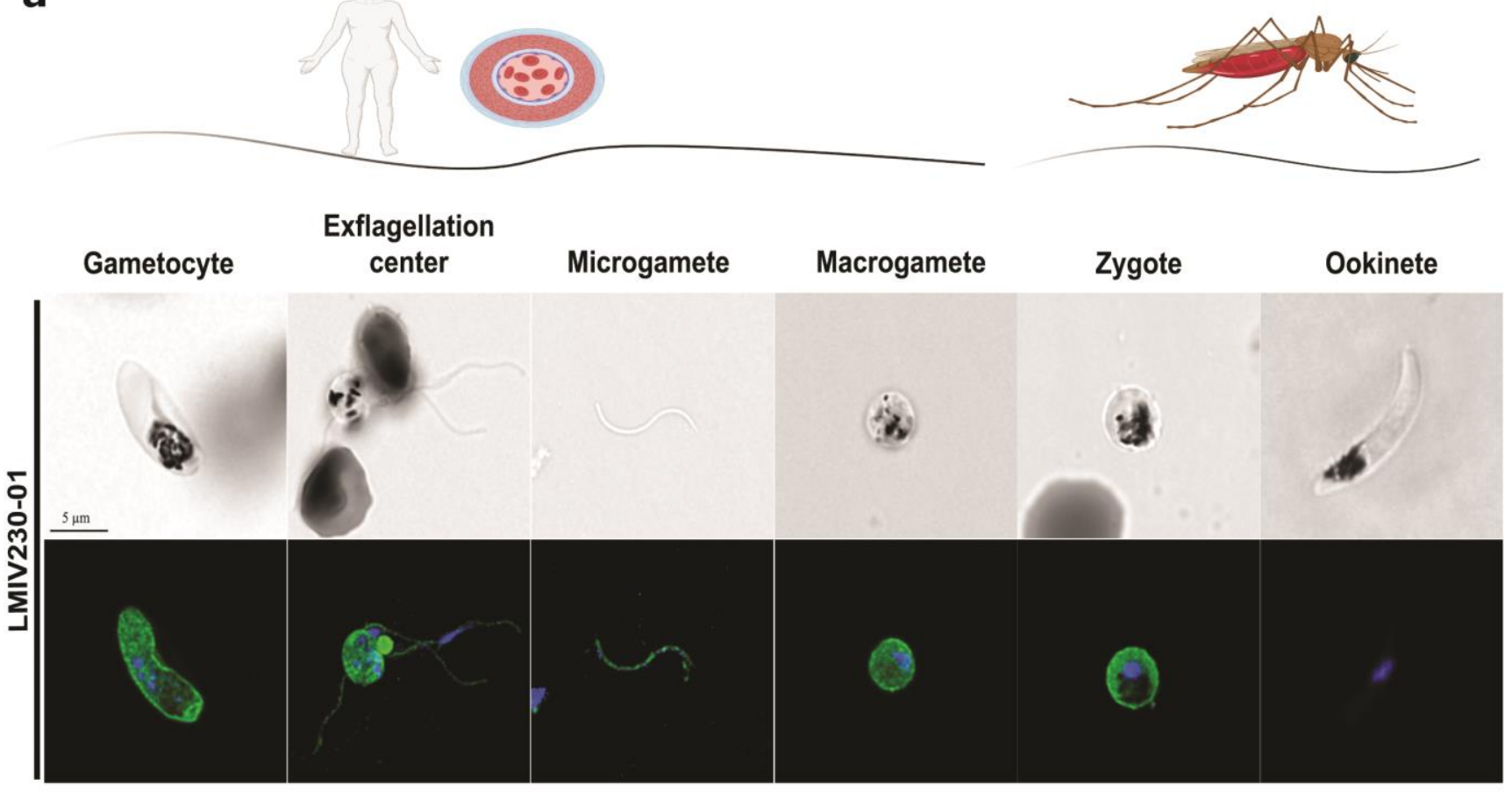

b

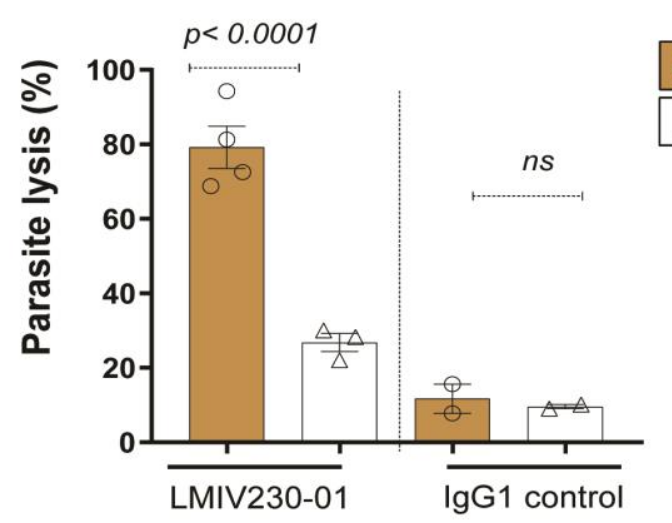

d

LMIV230-01

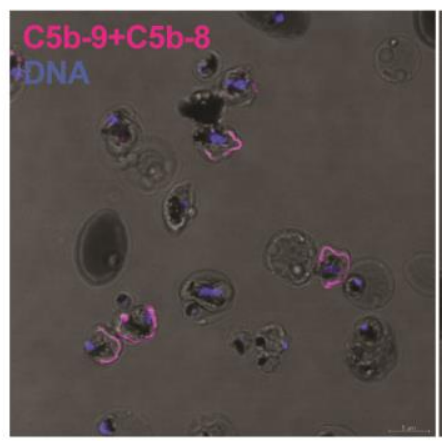

Intact serum

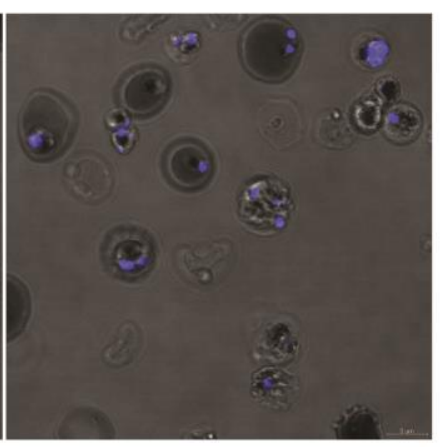

HI serum

C

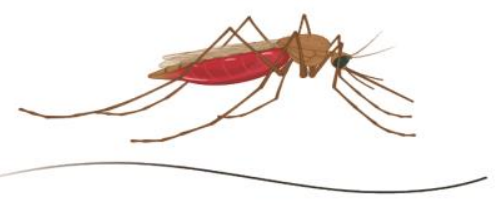

Zygote

SMFA

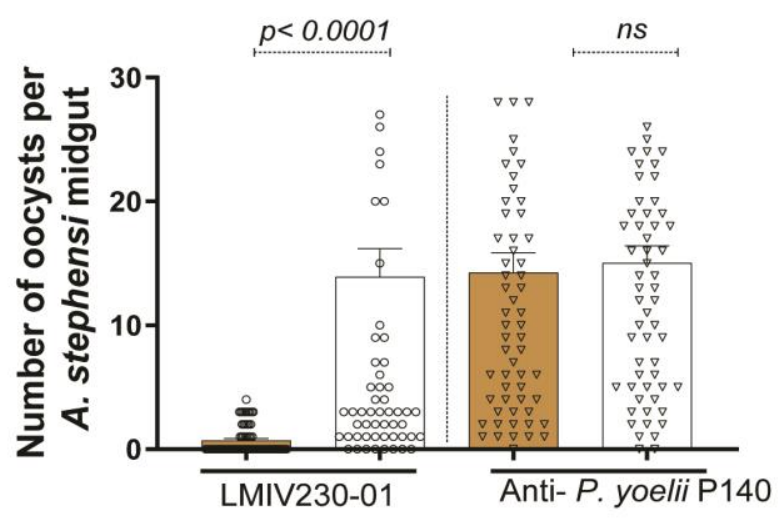

IgG1 control

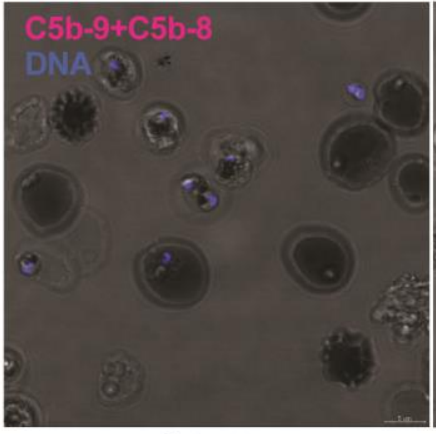

Intact serum

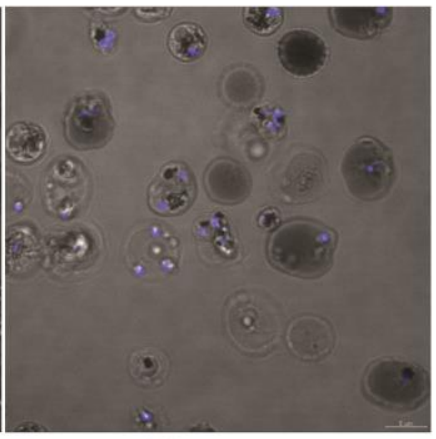

HI serum 
Fig. 2| LMIV230-01 binds to multiple parasite stages and its activity is complement-dependent. a, LMIV230-01 strongly binds to permeabilized gametocytes, gametes and zygotes and does not bind to ookinetes. Parasites were fixed and permeabilized, and $7.5 \mu \mathrm{g} / \mathrm{mL}$ of antibody was used to stain the different parasite stages. Scale bars: $5 \mu \mathrm{M}$. b, In vitro parasite lysis by LMIV230-01 is complementdependent. Samples were tested in two independent assays, using two different parasite cultures c, Functional activity of LMIV230-01 is also complement-dependent in vivo (SMFA with mosquitoes). Data from three independent SMFA assays. $\mathrm{N} \geq 20$ mosquitos per assay. Oocyst averages in the control mosquitoes (fed with lgG1 targeting $P$. yoelli P140) for each of the experiments were: exp. 1= 4.55; exp. $2=20.50$, exp. $3=5.86$. Data obtained from mosquitoes fed with LMIV230-01 at $1000 \mu \mathrm{g} / \mathrm{mL}$ with intact sera were also used to generate figure $1 \mathrm{f}$. Values are shown as mean \pm s.e.m. One-Way ANOVA and Turkey's multiple comparisons test were used to compare the different groups d, Live imaging of $P$. falciparum NF54 female gametes incubated with LMIV230-01 in the presence of intact serum from a healthy donor revealed surface-deposited MAC (membrane attack complex) using anti-C5b-9+C5b-8 antibody (magenta color). MAC deposition was not detected in the presence of heat-inactivated $(\mathrm{HI})$ serum. Scale bars: $5 \mu \mathrm{M}$.

To assess whether LMIV230-01 would also bind to other $P$. falciparum strains, we prepared gametocytes from a culture-adapted Malian isolate ${ }^{20}$ and from St. Lucia strain (originally from El Salvador) ${ }^{21}$. LMIV230-01 labelled in vitro-induced gametes from both strains (Fig. $3 a$ and b). Induction of gamete stage from the newly characterized Malian isolate was confirmed using a murine anti-Pfs48/45 mAb (Fig. 3c). LMIV230-01 fixed complement on the gamete surface of both strains, confirming that the antibody is functional against heterologous parasites (Figs. $3 d$ and e).

To assess the abundance of antibodies that share paratope specificity with LMIV230-01, we developed an ELISA assay to demonstrate the competition between post-vaccination sera (tested at a 1:2500 dilution) and LMIV230-01 for binding the vaccine antigen (Figure 3f). Among subjects who received the vaccine, levels of competing antibody ranged from $\sim 10-70 \%$ displacement of Pfs230D1 binding to LMIV230-01, with a normal distribution confirmed by Shapiro-Wilk test $(p=0.52)$ (Figure $3 g)$. Levels of competition strongly correlated with total Pfs230D1 lgG titers in sera (Spearman's rho $=0.604, p<0.0001$ ) (Figure 3h). Increasing levels of competing 
221 antibody also corresponded to serum functional activity measured by SMFA. Because 222 serum TRA levels of vaccines were high with minimal variability ranging from 95-100\% 223 (Extended Data Figure 14), our primary correlation analysis used TBA (Transmission 224 Blocking Activity) which indicates the \% reduction in the proportion of infected 225 mosquitoes, a high bar for TBV activity generally seen only when TRA is very high. 226 Correlation analyses showed that \% serum competition was related to TBA 227 (Spearman's rho $=0.324, p=0.05)$ (Figure 3i), suggesting that antibodies that compete 228 for the LMIV230-01 epitope play an important role in serum functional activity. This 229 result, however, does not exclude the possible role of antibodies that do not compete 230 with LMIV230-01 in mediating vaccine activity, and notably some sera with high TBA 231 demonstrated low levels of LMIV230-01 competing antibodies. 
a

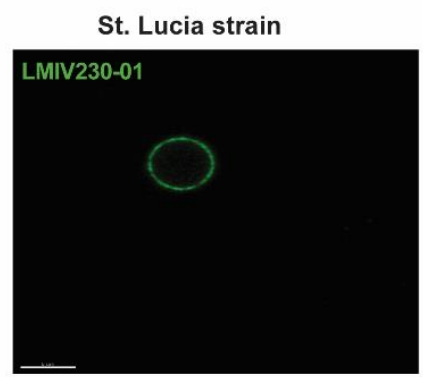

b

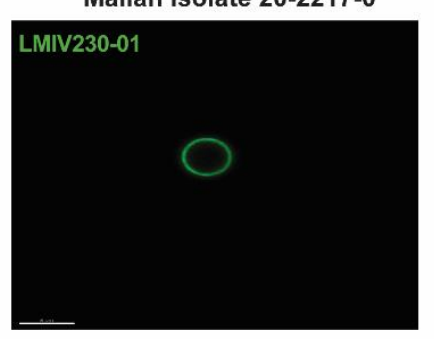

d

St. Lucia strain

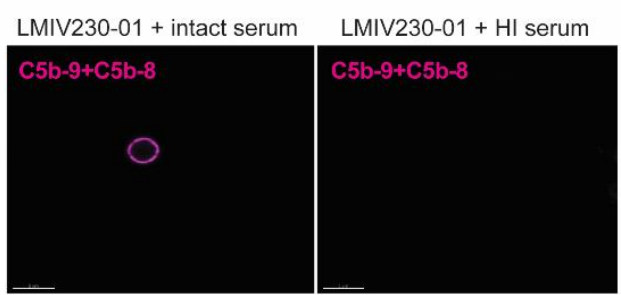

f
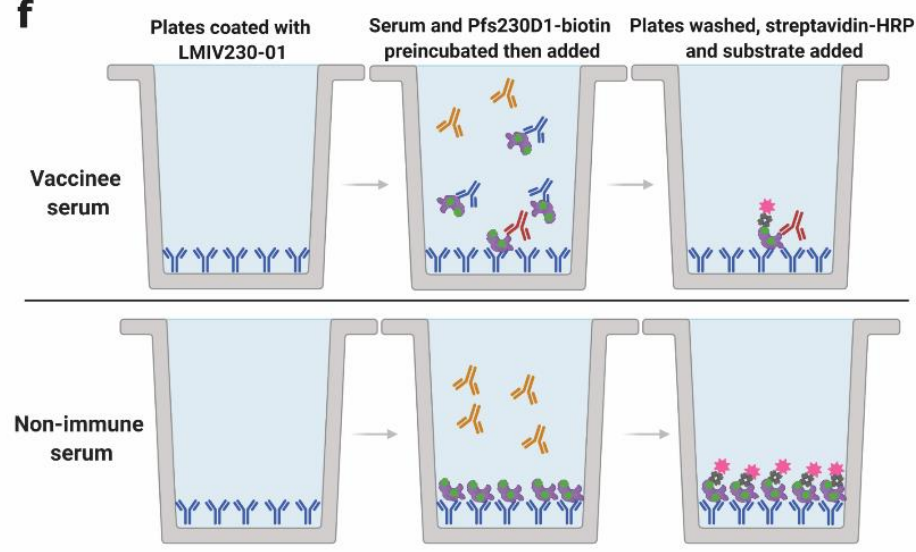

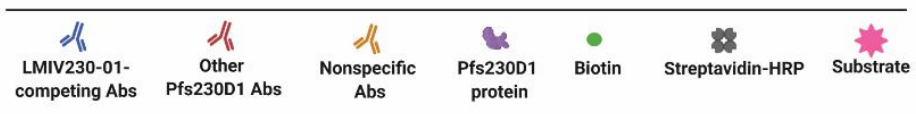

Malian isolate $20-2217-0$

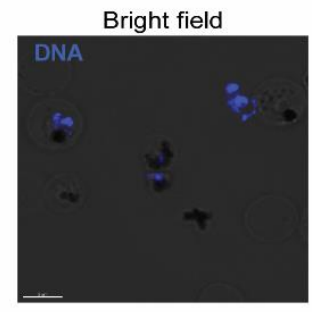

LMIV230-01

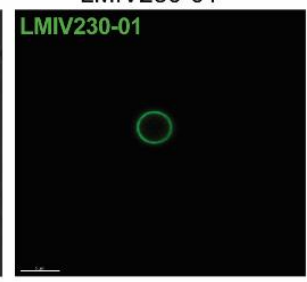

Merged
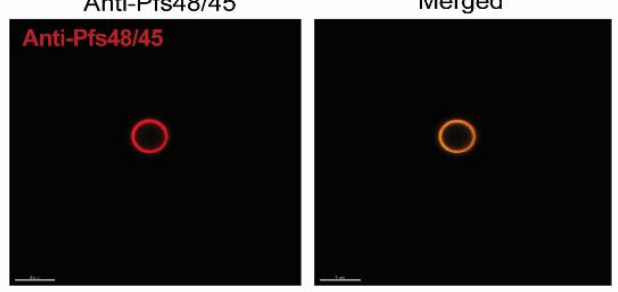

e

Malian isolate $\mathbf{2 0 - 2 2 1 7 - 0}$

LMIV230-01 + intact serum LMIV230-01 + HI serum

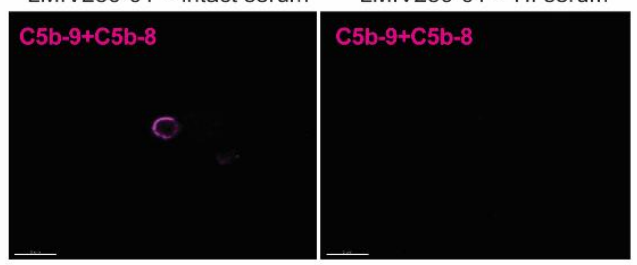

g

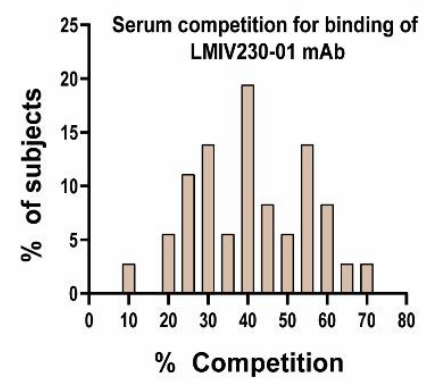

h

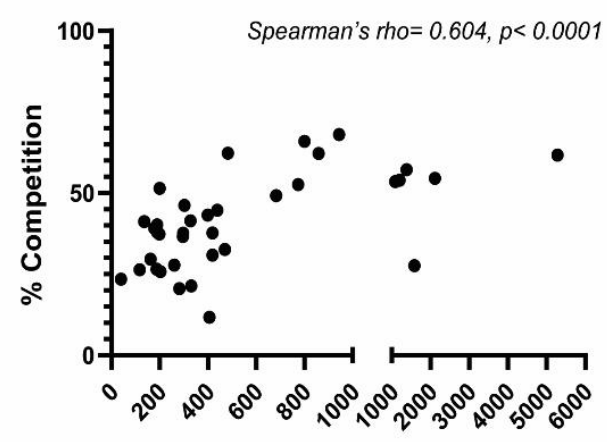

Pfs230D1 total IgG i

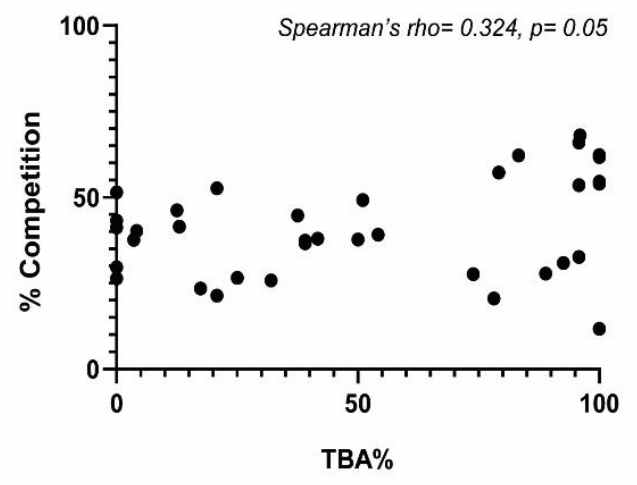


Figure 3| LMIV230-01 binds to heterologous $P$. falciparum strains and antisera from Pfs230D1 vaccinees vary widely in levels of antibody that compete with LMIV230-01 for binding. a, LMIV23001 bound to gametes of St. Lucia parasite strain and $\mathbf{b}$, of an isolate obtained from a Malian adult and adapted to culture. c, Murine anti-48/45 mAb confirms formation of gametes by Malian isolate and its signal colocalizes with LMIV230-01. "Merged" refers to combination of green and red channels. d, Membrane attack complex forms on gametes of St. Lucia strain and $\mathbf{e}$, of a Malian isolate incubated with LMIV230-01 in the presence of intact but not heat-inactivated serum. Scale bars for all images in this panel: $5 \mu \mathrm{M}$. f, Cartoon schematizing LMIV230-01 competition ELISA assay. g, Distribution of serum antibody levels that compete with LMIV230-01 for binding to PfS230D1 in 36 subjects who received Pfs230D1-EPA vaccine. Values displayed represent mean from three independent experiments. $\mathbf{h}$, Relationship of LMIV230-01-competing antibody levels to total Pfs230D1 antibody titers, or $\mathbf{i}$, to serum functional activity (TBA, transmission blocking activity) measured by SMFA.

Altogether, our data confirm that vaccination with TBV can elicit strong of impairing fertilization in the mosquito. Due to its complex domains and repeating motifs with numerous disulfide bonds, expression of full length Pfs230 has been difficult $^{22,23}$. Preclinical studies of Pfs230 fragments have shown that immunization with recombinant domain 1 of Pfs230 (Pfs230D1), but not other domains, induces strong functional TRA in SMFA $3,22,24$. 


\section{ACKNOWLEDGMENTS}

This work was funded by the Intramural Research Program of the National Institute of Allergy and Infectious Diseases, National Institutes of Health. Calvin Eigsti provided support for single cell sorting; Sundar Ganesan for live cell imaging; Ashley McCormack and Emily Higbee for Standard Membrane Feeding Assays; and J. Patrick Gorres for proofreading and editing this manuscript. We are thankful to Jillian Neal and Robert Morrison for determining the Pfs230D1 sequence for the Malian P. falciparum isolate. JT and JDG were supported by the Swiss National Science Foundation (AmbizioneSCORE fellowship to JT: PZ00P3_161147; PZ00P3_183777). We thank the staff members of GM/CA beamline at the Advanced Photon Source, Argonne National Laboratory for beamline support.

\section{AUTHOR CONTRIBUTIONS}

C.H.C. and P.E.D. conceived the single B cell sorting of Pfs230D1-specific B cells, V gene repertoire analyses, antibody generation, conventional and competitive ELISAs, Western blot, microscopy-based binding assays and in vitro and in vivo functional characterization of mAbs. W.K.T. and N.H.T. conceived the epitope binning and biophysical studies. C.H.C, W.K.T., N.H.T and P.E.D conceived the analysis of polymorphisms. C.H.C., W.K.T., M.B., J.R., A.S., T.A.S., W.P., X.H., B.B., O.M., B. J, M.S. and N.D.S. performed the experiments. M.E., C.H.C., and J.D.G. performed bioinformatic analyses. N.J.M., K.R., V.N., R.H., R.S. and D.N. generated recombinant Pfs230D1. I.S., J.J.T., J.V.R., J.T., J.H., M.B.S, J.R., N.H.T. and P.E.D. supervised the 
280

281

282

283

284

285

286

287

288

289

290

291

292

293

294

295

296

297

298

299

300

301

302

303

experiments and interpreted the data. C.H.C., W.K.T., N.H.T. and P.E.D. wrote the manuscript, with input from all authors.

\section{COMPETING INTERESTS}

M.B.S, W.P., X.H., B.B., and M.E. declare competing financial interests as all are employees of iRepertoire Inc., and J.H. is co-founder and CEO. J.D.G. is an employee of Alchemab Therapeutics Limited.

\section{CODE AVAILABILITY}

Code is available on request from the corresponding author.

9

0

1

2

3

4

96

97

99

300

301

302




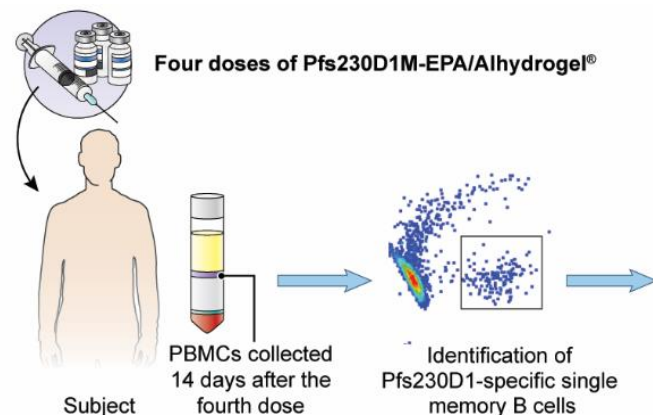

Subject fourth dose memory B cells
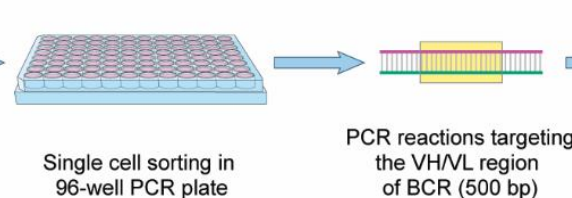
96-well PCR plate the VHNL region

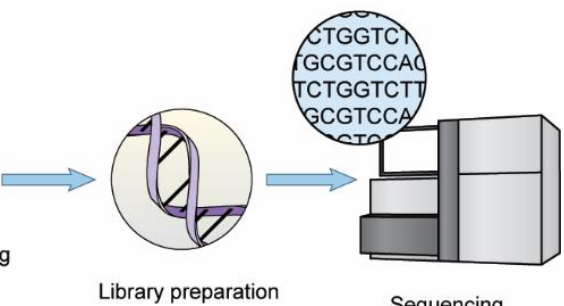

Library preparation

Sequencing

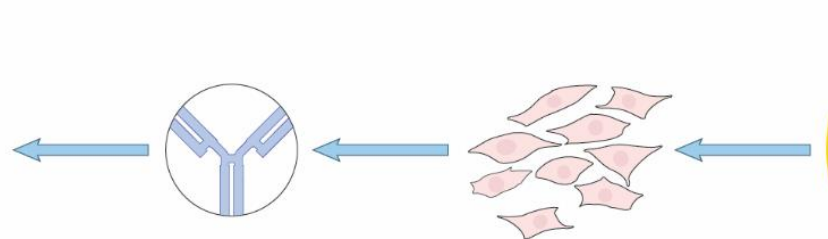

Pfs230D1 mAbs purification

Expression in 293 HEK cells

Binding, functional and structural analyses

Extended Data Fig. 1| Experimental pipeline. Pfs230D1-specific single B cells were sorted from PBMCs of eight Malian adults who had been immunized with four doses of $40 \mu \mathrm{g}$ of Pfs230D1-EPA/Alhydrogel囚. After extraction of single B cells, a 500 bp fragment of the BCR variable regions of $V H / V L$ were amplified and sequenced. Matched VH/VL pairs that were identified in more than one B cell were preferentially selected for cloning in an IgG1 vector for expression in 293 HEK cells and subsequent analyses. 


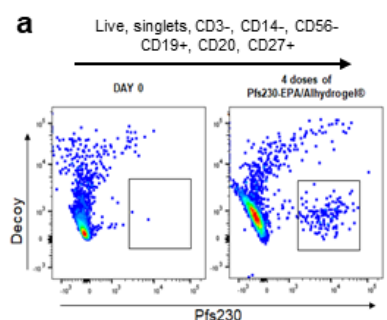

b

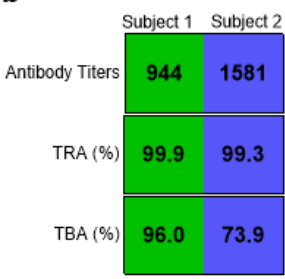

e
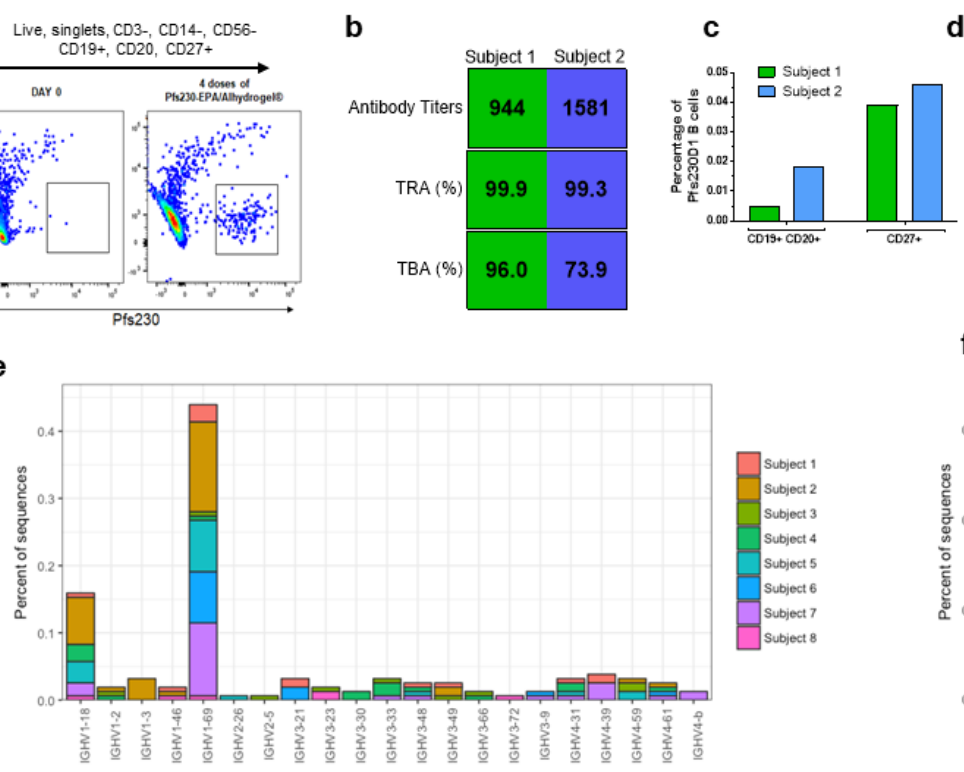

d

\begin{tabular}{c|c|c|c|c|c|}
\hline Gene & mAb & $\begin{array}{c}\text { VH and } \\
\text { VLIK }\end{array}$ & CDR1 & CDR2 & CDR3 \\
\hline $\begin{array}{c}\text { Heavy } \\
\text { chain }\end{array}$ & LMIV230-01 & 1 GHV1-69 & GGTFNTYA & IIPFQDRG & ARESGRAVADR \\
& LMIV230-02 & 1 GHV1-69 & GGTFNSYG & IIPSFGTA & AGSSWSSGTYYGRSAY \\
\hline $\begin{array}{l}\text { Light } \\
\text { chain }\end{array}$ & LMIV230-01 & 1 GKV1-5 & HSISSW & KAS & QQYKSYPWT \\
& LMIV230-02 & $1 G K V 4-1$ & QSVLYSSNNKNY & WAS & QQYYSTPYS \\
\hline
\end{tabular}

f

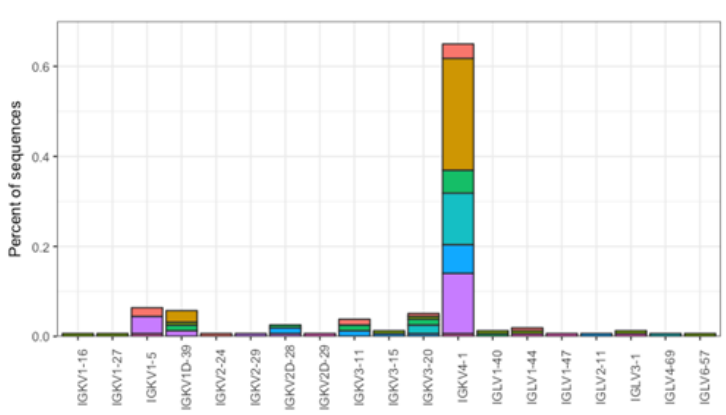

Extended Data Fig. 2| Pfs230D1-specific mAbs belong to the same heavy chain germline subgroup but differ for kappa chain. a, Sorted memory B cells were gated as live, single cells, excluded for CD3, CD14 and CD56, and gated on CD19+, CD20+, CD27+ cells. Then, a tetramer approach was used to select antigen-specific cells and reduce nonspecific binding. Cells binding to the decoy tetramer (BSA) were excluded and only those binding to Pfs230D1 were selected for sorting. $\mathbf{b}$, Serum from each subject was used to measured antibody titers against Pfs230D1 and functional activity to reduce oocyst burden in Standard Membrane Feeding Assays (SMFA). TRA = Transmission Reducing Activity measured as the reduction in average oocyst count; TBA $=$ Transmission Blocking Activity measured as the reduction in the proportion of infected mosquitoes. c, Proportion of memory B cells for each subject that are Pfs230D1specific. d, Complementarity-determining regions (CDRs) of each sequence selected for mAb expression. e, IGKV4-1 germline (gene sequence in LMIV230-02) was the most frequent for the kappa chain genes. IGKV1-5 germline (gene sequence in LMIV230-01) was found in only three subjects $\mathbf{f}$, Sequences related to germline 1-69 of the IGHV gene were the most frequently elicited in response to the vaccination. 


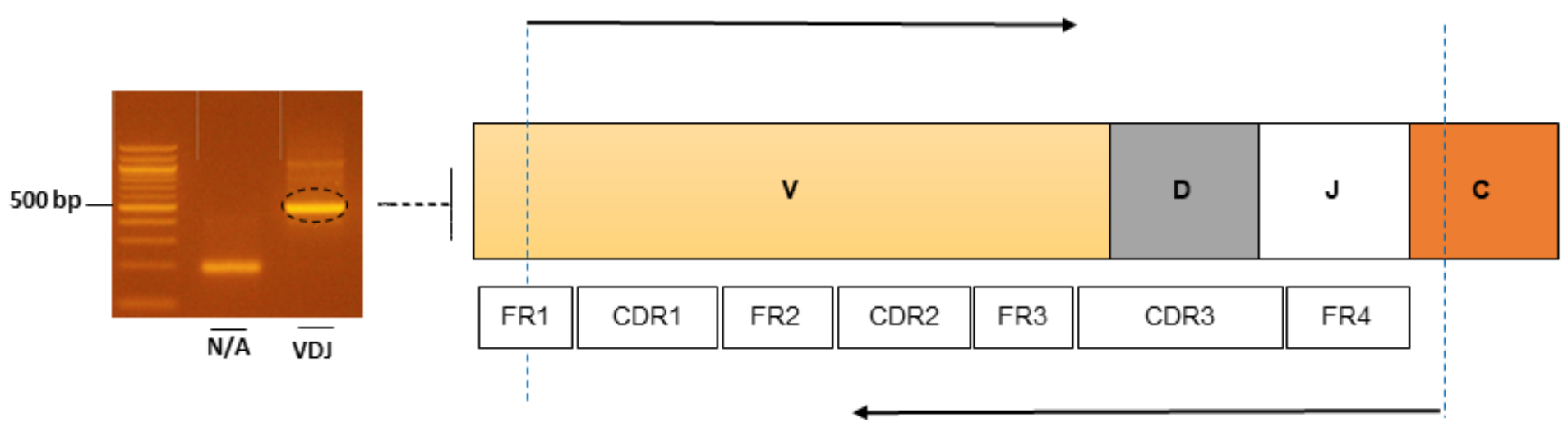

331 Extended Data Fig. 3| Amplification of V(D)J region. 500 bp fragment amplified from cDNA of sorted 332 Pfs230D1-specific single $B$ cell. This fragment was obtained using primers targeting the $V(D) J$ region 333 (iRepertoire Inc.). 

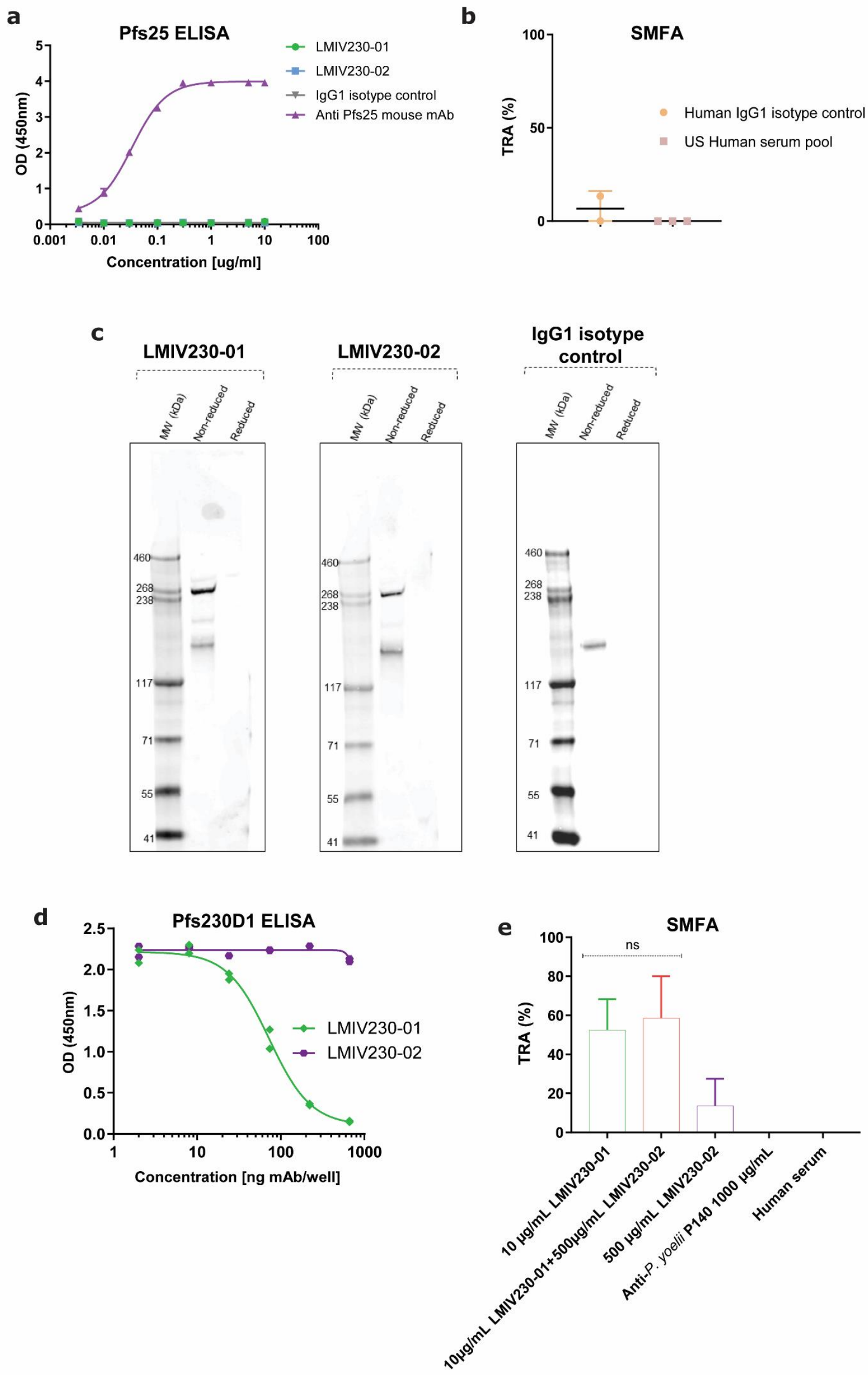
Extended Data Fig.5| Additional binding and functional characterization of LMIV230-01 and -02. a, Both mAbs failed to bind to the ookinete protein Pfs25. b. Additional controls for the Standard Membrane Feeding Assay (SMFA). Human IgG1 isotype control was expressed using the same conditions as LMIV230-01 and -02 and was used in this assay at $1000 \mu \mathrm{g} / \mathrm{mL}$. Sixty microliters of undiluted human pooled serum obtained from US healthy donors were used as additional control. Values are shown as mean \pm s.e.m. c, Full depiction of the Western blot gel displayed in Fig. $1 \mathrm{~g} . \mathbf{d}$, The two mAbs do not compete for the same epitope in the recombinant Pfs230D1 protein, since unlabelled LMIV230-01 blocks binding of LMIV-230-01-HRP to immobilized Pfs230D1 but LMIV230-02 does not. e, Combination of LMIV230-01 and LMIV230-02 did not increase functional activity over LMIV230-01 alone. Control mosquitoes were fed with mouse IgG1 mAb targeting $P$. yoelii P140 protein, or with non-immune human serum.

351

352

353

354

355

356

357

358

359

360 
a

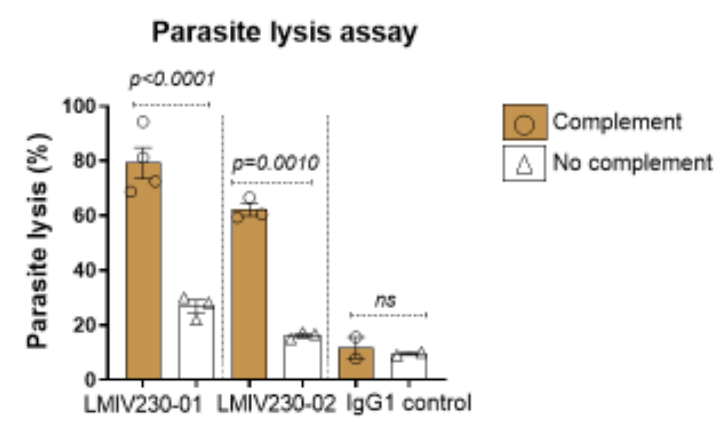

b

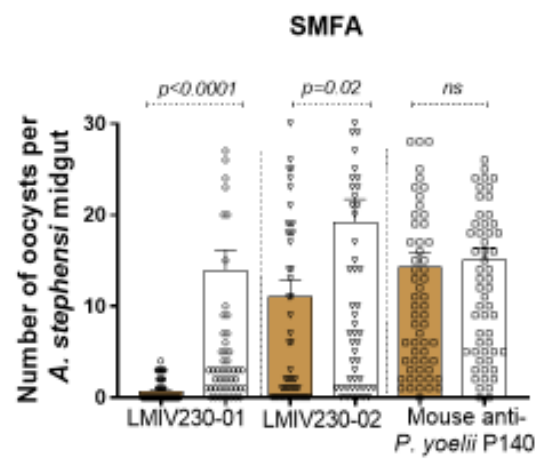

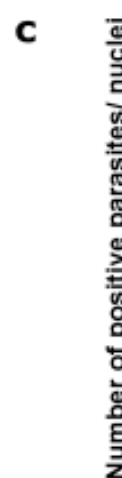

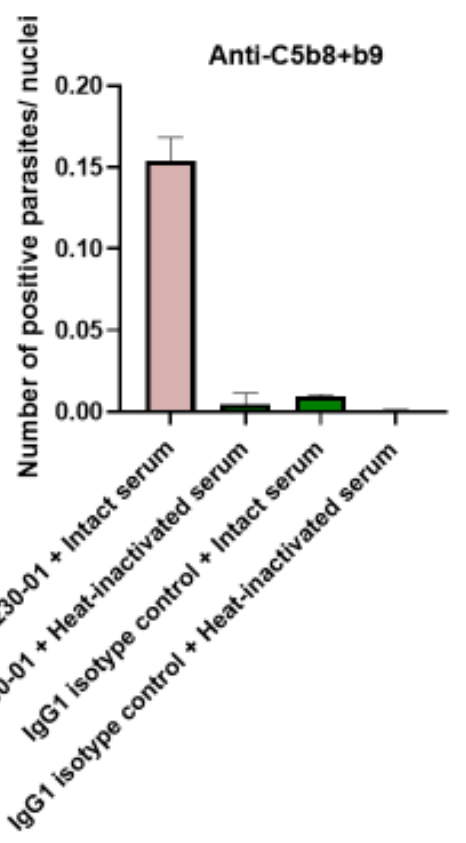

362

\section{3}

Extended Data Fig.7| Pfs230 mAbs activity is complement-dependent and LMIV230-01 competing antibodies are acquired at varying levels by vaccinees. a, Activity of LMIV230-01 and LMIV230-02 is complement-dependent in the vitro lysis assay and $\mathbf{b}$, in the vivo mosquito feeding assay. $\mathbf{c}$, Membrane attack complexes (MAC) on parasites were detected using an Alexa 488-labeled antibody that recognizes the assembled MAC complex (anti C5b-9+ C5b-8). Gametes incubated with LMIV230-01 and intact serum produced MAC-positive parasites. Heat-inactivating serum to degrade the heat-labile components of the complement pathway eliminated deposition of MAC on gametes. MAC-positive $P$. falciparum strain NF54 gametes were enumerated in a large, tiled confocal image and normalized to the number of Hoechst-stained nuclei. 


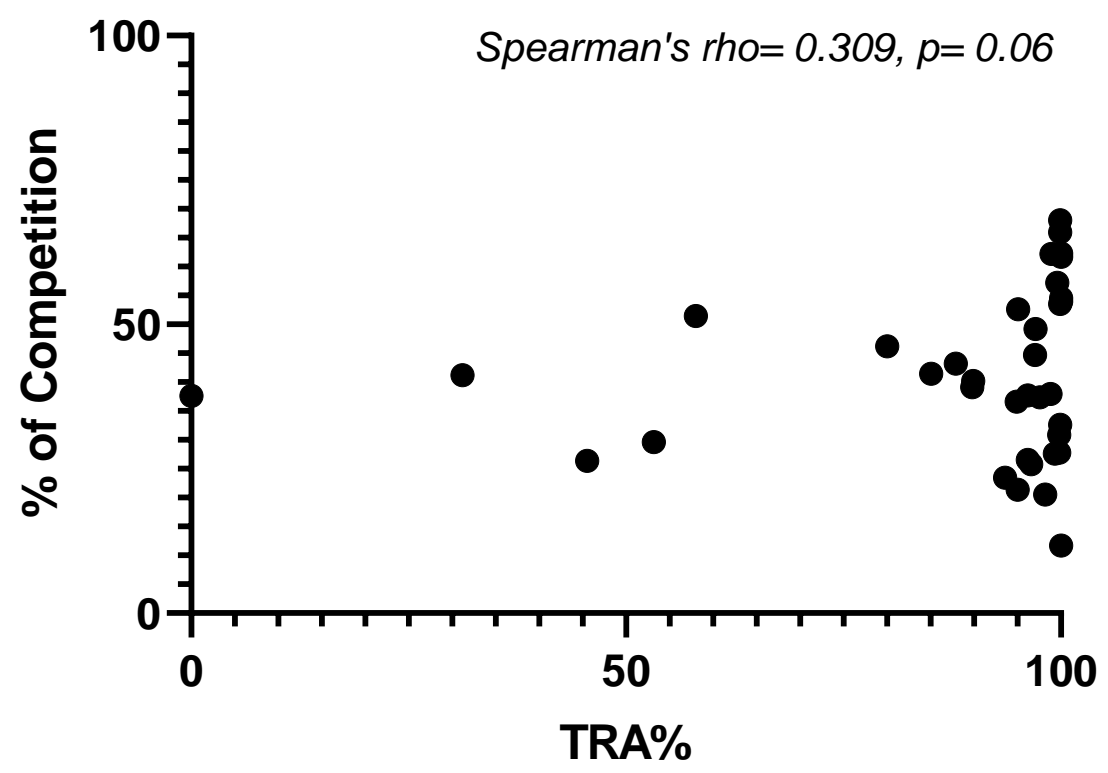
Transmission-Reducing Activity (TRA) measured in SMFA. 
400

\begin{tabular}{cccc}
\hline $\begin{array}{c}\text { Subject } \\
\text { ID }\end{array}$ & $\begin{array}{c}\text { Antibody } \\
\text { titers }\end{array}$ & TRA (\%) & TBA (\%) \\
\hline 1 & 944 & 99.9 & 96 \\
2 & 1581 & 99.3 & 73.9 \\
3 & 2115 & 100 & 100 \\
4 & 1382 & 99.6 & 79.2 \\
5 & 2100 & 100 & 100 \\
6 & 5277 & 100 & 100 \\
7 & 800 & 99.9 & 95.8 \\
8 & 774 & 95.1 & 20.8 \\
& & &
\end{tabular}

402

403

404

405

406

407

408

409

410

411

412

413

414
Extended Data Table 1- Antibody titers and functional activity of sera from the eight subjects whose sequences were analyzed in this study. TRA $=$ Transmission-reducing activity. TBA=Transmission blocking activity.

\begin{tabular}{|c|c|c|c|c|}
\hline & $\begin{array}{c}K_{D} \\
\left(\times 10^{-10} \pm \text { SEM M }\right)\end{array}$ & $\begin{array}{c}k_{a} \\
\left(x 10^{5} \pm \text { SEM 1/Ms }\right)\end{array}$ & $\begin{array}{c}k_{\text {dis }} \\
\left(\times 10^{-4} \pm \text { SEM 1/s) }\right.\end{array}$ & $\mathbf{N}$ \\
\hline \multicolumn{5}{|l|}{ LMIV230-01 } \\
\hline Biological Replicate 1 & $1.58 \pm 0.77$ & $1.71 \pm 0.06$ & $0.28 \pm 0.15$ & 3 \\
\hline Biological Replicate 2 & $2.06 \pm 0.99$ & $1.80 \pm 0.04$ & $0.37 \pm 0.18$ & 3 \\
\hline \multicolumn{5}{|l|}{ LMIV230-02 } \\
\hline Biological Replicate 1 & $6.36 \pm 0.24$ & $7.67 \pm 0.21$ & $4.87 \pm 0.06$ & 3 \\
\hline Biological Replicate 2 & $4.27 \pm 0.22$ & $6.37 \pm 0.13$ & $2.71 \pm 0.10$ & 3 \\
\hline
\end{tabular}

Extended Data Table 2- Binding of mAbs LMIV230-01 and LMIV230-02 to Pfs230D1 using Biolayer Interferometry. Binding data for each mAb was fitted using a 1:1 binding model. The averages for two biological replicates, composed of three technical replicates each, are shown for both mAbs. 


\section{REFERENCES}

418 1. Eksi, S., et al. Malaria transmission-blocking antigen, Pfs230, mediates human red blood cell binding to

exflagellating male parasites and oocyst production. Mol Microbiol 61, 991-998 (2006).

2. Williamson, K.C., Keister, D.B., Muratova, O. \& Kaslow, D.C. Recombinant Pfs230, a Plasmodium falciparum gametocyte protein, induces antisera that reduce the infectivity of Plasmodium falciparum to mosquitoes. Mol Biochem Parasitol 75, 33-42 (1995).

3. Tachibana, M., et al. Identification of domains within Pfs230 that elicit transmission blocking antibody responses. Vaccine 37, 1799-1806 (2019).

4. Triller, G., et al. Natural Parasite Exposure Induces Protective Human Anti-Malarial Antibodies. Immunity 47, 1197-1209 e1110 (2017).

5. Kisalu, N.K., et al. A human monoclonal antibody prevents malaria infection by targeting a new site of vulnerability on the parasite. Nat Med 24, 408-416 (2018).

6. Alanine, D.G.W., et al. Human Antibodies that Slow Erythrocyte Invasion Potentiate Malaria-Neutralizing Antibodies. Cell 178, 216-228 e221 (2019).

7. Rappuoli, R., Bottomley, M.J., D'Oro, U., Finco, O. \& De Gregorio, E. Reverse vaccinology 2.0: Human immunology instructs vaccine antigen design. J Exp Med 213, 469-481 (2016).

8. Tan, J., et al. A LAIR1 insertion generates broadly reactive antibodies against malaria variant antigens. Nature 529, 105-109 (2016).

9. Watson, C.T., et al. Complete haplotype sequence of the human immunoglobulin heavy-chain variable, diversity, and joining genes and characterization of allelic and copy-number variation. Am J Hum Genet $\mathbf{9 2}$, 530-546 (2013).

10. Avnir, Y., et al. Molecular signatures of hemagglutinin stem-directed heterosubtypic human neutralizing antibodies against influenza A viruses. PLoS Pathog 10, e1004103 (2014).

11. Avnir, Y., et al. IGHV1-69 polymorphism modulates anti-influenza antibody repertoires, correlates with IGHV utilization shifts and varies by ethnicity. Sci Rep 6, 20842 (2016).

12. Lensen, A., et al. Measurement by membrane feeding of reduction in Plasmodium falciparum transmission induced by endemic sera. Trans R Soc Trop Med Hyg 90, 20-22 (1996).

13. Miura, K., et al. Transmission-blocking activity is determined by transmission-reducing activity and number of control oocysts in Plasmodium falciparum standard membrane-feeding assay. Vaccine 34, 4145-4151 (2016).

14. Doolan, K.M. \& Colby, D.W. Conformation-dependent epitopes recognized by prion protein antibodies probed using mutational scanning and deep sequencing. J Mol Biol 427, 328-340 (2015).

15. Verrier, F.C., et al. Antibodies to several conformation-dependent epitopes of gp120/gp41 inhibit CCR-5dependent cell-to-cell fusion mediated by the native envelope glycoprotein of a primary macrophage-tropic HIV-1 isolate. Proc Natl Acad Sci U S A 94, 9326-9331 (1997).

16. Outchkourov, N., et al. Epitope analysis of the malaria surface antigen pfs48/45 identifies a subdomain that elicits transmission blocking antibodies. J Biol Chem 282, 17148-17156 (2007).

17. Healer, J., et al. Complement-mediated lysis of Plasmodium falciparum gametes by malaria-immune human sera is associated with antibodies to the gamete surface antigen Pfs230. Infect Immun 65, 3017-3023 (1997).

18. Tegla, C.A., et al. Membrane attack by complement: the assembly and biology of terminal complement complexes. Immunol Res 51, 45-60 (2011).

19. Bayly-Jones, C., Bubeck, D. \& Dunstone, M.A. The mystery behind membrane insertion: a review of the complement membrane attack complex. Philos Trans R Soc Lond B Biol Sci 372(2017).

20. Doritchamou, J.Y.A., et al. Placental malaria vaccine candidate antigen VAR2CSA displays atypical domain architecture in some Plasmodium falciparum strains. Commun Biol 2, 457 (2019).

21. Collins, W.E., Warren, M., Skinner, J.C., Richardson, B.B. \& Kearse, T.S. Infectivity of the Santa Lucia (EI Salvador) strain of Plasmodium falciparum to different anophelines. J Parasitol 63, 57-61 (1977).

22. MacDonald, N.J., et al. Structural and Immunological Characterization of Recombinant 6-Cysteine Domains of the Plasmodium falciparum Sexual Stage Protein Pfs230. J Biol Chem 291, 19913-19922 (2016).

23. Farrance, C.E., et al. A plant-produced Pfs230 vaccine candidate blocks transmission of Plasmodium falciparum. Clin Vaccine Immunol 18, 1351-1357 (2011).

24. Singh, S.K., et al. Pfs230 and Pfs48/45 Fusion Proteins Elicit Strong Transmission-Blocking Antibody Responses Against Plasmodium falciparum. Front Immunol 10, 1256 (2019). 
471

472

473

474

475

476

477

478

479

480

481
25. Tonkin, M.L., et al. Structural and biochemical characterization of Plasmodium falciparum 12 (Pf12) reveals a unique interdomain organization and the potential for an antiparallel arrangement with Pf41. J Biol Chem 288, 12805-12817 (2013).

26. Parker, M.L., Peng, F. \& Boulanger, M.J. The Structure of Plasmodium falciparum Blood-Stage 6-Cys Protein Pf41 Reveals an Unexpected Intra-Domain Insertion Required for Pf12 Coordination. PLoS One 10, e0139407 (2015).

27. Kundu, P., et al. Structural delineation of potent transmission-blocking epitope I on malaria antigen Pfs48/45. Nat Commun 9, 4458 (2018).

28. Holm, L. \& Rosenstrom, P. Dali server: conservation mapping in 3D. Nucleic Acids Res 38, W545-549 (2010). 